\title{
Muerte encefálica, bioética y trasplante de órganos
}

\author{
Grupo de Estudios sobre Muerte Encefálica, de las \\ Sociedades Chilenas de N efrología y de Trasplante
}

\section{Brain death, bioethics and organ transplantation}

The concept of death has evolved medically, legally and culturally since the introduction of life support technologies in the middle of the 20th century. The traditional cardiopulmonary and the new neurologically based brain death criterions of death are examined. We conclude that brain death, defined as total and irreversible loss of function of the whole brain, fulfills better "the permanent cessation of functioning of the organism as a whole" definition of death. Brain death diagnosis, based on standard neurologic clinical examination performed accurately, is unequivocal. Transplantation medicine, mostly based on organ donation of brain dead people, has become a routine and universally accepted therapeutic intervention nowadays, which benefits many people. Ethics foundations of organ transplantation are reviewed. Even though brain death and organ donation are widely accepted in medical, legal, religious and public opinion today, the whole society and medical community need to be further educated about these matters, so that unavoidable changes of traditional concepts might be better understood. Permanent education should be the best way to dissipate social fears and distrust towards organ donation and brain death (Rev Méd Chile 2004; 132: 109-18).

(Key Words: Brain death; Death; Ethics, medical; Transplantation)

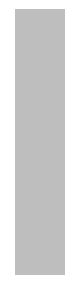

\begin{abstract}
Documento elaborado por el Grupo de Estudios Sobre Muerte Encefálica de las Sociedades Chilenas de Nefrología y de Trasplante. Aprobado para publicación el 21 de octubre de 2003.

Integrantes de Grupo de Estudios Sobre Muerte Encefálica: Drs. Juan Carlos Flores H (Servicio de Nefrología, Hospital Militar, Santiago), Manuel Pérez F (Servicio de Neurología, Clínica Las Condes, Santiago), Sergio Thambo B (Facultad de Medicina y Hospital Clínico, Universidad de Chile), Andrés Valdivieso D (Facultad de Medicina y Hospital Clínico, Pontificia Universidad Católica de Chile)
\end{abstract}

$\mathrm{E}^{1}$ concepto de muerte ha evolucionado médica, legal y culturalmente en una era de medicina de alta tecnología. Hasta recientemente la mente y el cuerpo cesaban de funcionar al mismo tiempo y esta muerte era generalmente vista como un solo fenómeno. En el mundo moderno las definiciones de muerte con criterio neurológico o cerebral surgieron como conse- cuencia de la transformación tecnológica de la muerte como un fenómeno unitario.

La definición y determinación de la muerte han sido una historia de incertidumbre, siendo pocas veces una tarea fácil y nunca una empresa segura $^{1}$. La naturaleza de la muerte es un problema antiguo; por más de veinticinco siglos el hombre se ha planteado interrogantes como: ¿qué 
es la muerte?, ¿cuándo morimos realmente? No hay una respuesta sencilla para estas preguntas ya que la muerte conlleva una serie de implicaciones éticas, religiosas, filosóficas, médicas y legales que hacen muy difícil su abordaje.

Los cambios producidos en el concepto de muerte interesan a la sociedad en general por la diversidad de las consecuencias mencionadas, y a la ciencia médica en particular porque a ella se le exige precisión y exactitud sobre el momento de la muerte del ser humano. Los médicos deben estar conscientes que el tema de la muerte es ineludible y atraviesa todas las áreas de la medicina, desde la medicina primaria y familiar hasta la medicina terciaria que se realiza en las unidades de cuidados intensivos y en las unidades de trasplante de órganos.

Nos proponemos en este artículo contribuir a un debate, en ocasiones apasionado, revisando los aspectos históricos, médicos, ético-morales, legales y culturales de la muerte y su evolución durante las décadas recientes. Esta revisión está dirigida principalmente a la comunidad médica, ya que en ella recae la gran responsabilidad de construir una medicina más humana y basada en los más altos valores éticos y morales en una época de vertiginosos cambios.

\section{EL CONCEPTO DE MUERTE: UNA PERSPECTIVA HISTÓRICA}

Desde la antigüedad hasta el siglo XX. Para los antiguos griegos, hace 25 siglos, el ser humano sintetizaba en su naturaleza la integración de tres tipos de funciones: 1) Las funciones naturales 0 vegetativas (nutrición, crecimiento, reproducción), con sede en el abdomen, 2) Las funciones vitales 0 animales (pulso y respiración), radicadas en el tórax, y 3) Las funciones intelectivas o espirituales (pensamiento y voluntad), con sede en el cráneo. De acuerdo a este pensamiento, los espíritus reunidos en el hombre eran tres, el natural, el vital y el espiritual. Para los griegos la muerte consistía de la pérdida del espíritu denominado vital, radicado principalmente en el corazón, de ahí que la muerte haya coincidido tradicionalmente con la ausencia de pulso y respiración espontáneas. El criterio griego de muerte es el cardiopulmonar.
Sin embargo, ellos se planteaban también la cuestión de qué sucedería si el espíritu intelectivo pereciera antes que el vital; por ejemplo, por una herida del cerebro. ¿Sería esto una muerte? Respondían afirmativamente a esta interrogante. Galeno decía que el proceso de morir puede seguir dos vías, una ascendente, que comienza por la pérdida del espíritu vital (muerte cardiorrespiratoria) y termina por la pérdida del espíritu intelectivo (muerte cerebral), y otra descendente, que se inicia con la pérdida del espíritu intelectivo y finaliza con el paro cardiorrespiratorio ${ }^{2}$.

En la tradición judía el signo principal de vida era la respiración. Maimónides, un célebre médico judío decía que si durante el examen ningún signo de respiración puede ser detectado en la nariz, la víctima debe ser dejada donde se halle, pues ya está muerta».

Durante el siglo XVIII comienza a difundirse la idea de que ningún signo, ni siquiera el cese de la respiración y del pulso cardíaco, podía aclarar el diagnóstico de muerte excepto la aparición de los fenómenos de putrefacción. En la raíz de este pensamiento está lo que permitirá afirmar a Virchow, un siglo más tarde, que es la célula la unidad estructural y funcional más pequeña del ser humano y por tanto, la verdadera muerte es la muerte celular. Sólo la descomposición celular es verdadero signo de muerte. La muerte cardiopulmonar es sólo premonitoria. De ahí que las legislaciones empezaran a exigir un tiempo precautorio de $24 \mathrm{~h}$ antes del entierro del cadáver ${ }^{1,2}$.

A finales del siglo XVIII la formulación de la teoría de los tejidos plantea nuevos problemas. De acuerdo a ella el cuerpo humano está constituido por muchos diferentes tipos de tejidos, cada uno con su propia vitalidad y capacidad de respuesta a estímulos. En el ser humano, la muerte de cada órgano y tejido es un proceso asincrónico que puede durar días o semanas. Se plantea la cuestión del ¿cómo distinguir entre la muerte de un organismo individual complejo de las diversas partes que lo componen? Se inicia en esa época el conocimiento de la diferenciación en el sistema nervioso central de los diferentes estratos evolutivos: el paleoencéfalo, responsable de funciones vegetativas involuntarias, y el neoencéfalo o corteza cerebral, donde radica la vida de relación, la 
conciencia y la voluntad. Se establece el concepto que la diferencia entre la vida de un organismo y las vidas de sus partes, es la capacidad de integrar y coordinar esas partes en un todo, capacidad esta ejercida por el sistema nervioso ${ }^{1,2}$.

Aceptando la definición de muerte como una desorganización, la pérdida permanente del funcionamiento del organismo «como un todo», el cese de la actividad cardíaca y respiratoria era el mejor y más obvio criterio para determinar la frontera entre la vida y la muerte, ya que desde el momento en que se produce, el deterioro de las estructuras orgánicas del ser humano, incluido el sistema nervioso central, es rápido e irreversible.

Siglo XX. Durante siglos la determinación de la muerte se basó en la relativamente sencilla constatación del cese irreversible de la respiración y la circulación, sin las cuales era obvio que no se podía seguir viviendo. A partir de la segunda mitad del siglo XX, la introducción de los ventiladores mecánicos (desarrollados en concomitancia a las epidemias de polio de las décadas del 40 y 50), permitió mantener las funciones vitales en pacientes que habían sufrido la pérdida de la función cerebral.

Ya en 1959 Wertheimer, Jouvet y Descotes describen pacientes en coma y paro respiratorio tratados con ventilación artificial; denominan a estos cuadros «muerte del sistema nervioso»y discuten la justificación de desconectar a estos pacientes del respirador ${ }^{3}$. En ese mismo año Mollaret y Goulon, introducen el término coma sobrepasado (coma depassé) o irreversible, describiendo 23 pacientes comatosos sin reflejos de tronco cerebral, en apnea y con electroencefalograma plano ${ }^{4}$. Había nacido el concepto de muerte cerebral. En los años siguientes los pacientes y sus familias comenzaron a manifestar una alarma creciente respecto a que la tecnología médica podría sostener vitalmente por tiempo indeterminado a cuerpos de personas cuya existencia ya había finalizado.

Con el objeto de soslayar estas dudas y enfrentar estos problemas, en 1968 un comité ad hoc de la Escuela de Medicina de la Universidad de Harvard reexaminó la definición de muerte ${ }^{5}$. Este comité fue uno de los primeros en advocar una nueva definición de muerte basada en un criterio neurológico, estableciendo que los criterios clínicos que constituían el coma irreversible o muerte cerebral eran: una completa falta de respuesta y sensibilidad, la ausencia de movimiento y respiración espontánea, la ausencia de reflejos de tronco cerebral, y coma de causa identificable. En 1971, Mohandas y Chou describen el daño del tronco cerebral como el componente crítico y fundamental del daño cerebral severo ${ }^{6}$. Siguiendo este criterio, en 1976 la Conferencia de Colegios Médicos y sus Facultades del Reino Unido estableció, en lo que se conoce como Código del Reino Unido, que la muerte cerebral se define como la pérdida total e irreversible de la función del tronco encefálico ${ }^{7}$.

El siguiente hito en la historia del concepto de muerte cerebral lo estableció la Comisión del Presidente de Estados Unidos para el estudio de problemas éticos en medicina e investigación biomédica, que se reunió en 1981, y después de recibir los testimonios de gente de las áreas médica, filosófica, teológica y de la ley, acordó que un individuo puede ser declarado muerto sobre las bases de da cesación irreversible de las funciones respiratoria y circulatoria o la cesación irreversible de todas las funciones cerebrales incluyendo el tronco»?.

En la década del 80, siguiendo las recomendaciones de la Comisión Presidencial, también denominada Acta Uniforme para la Declaración de Muerte (UDDA), los 50 estados de Estados Unidos iniciaron el reemplazo de la definición cardiopulmonar de muerte por una que también incluía la cesación total e irreversible de la función cerebral. Desde entonces se ha ido produciendo un consenso gradual en la mayoría de los países, que han ido adoptando y perfeccionando en sus legislaciones este nuevo concepto de muerte, que ha perdurado tenazmente por más de 30 años aun enfrentando una crítica persistente.

\section{DEFINICIÓN Y CRITERIOS DE MUERTE}

Cuando se enfrenta el tema de la muerte, gran parte de la confusión se produce por la falta de 
una separación rigurosa y una formulación ordenada de tres elementos distintos:

1) La definición de muerte;

2) Los criterios para determinar que ella ha ocurrido; y

3) Los medios para probar que los criterios han sido satisfechos.

La definición es una tarea primariamente filosófica, la elección del criterio es primariamente médica y la selección de las pruebas es una materia exclusivamente médica ${ }^{9}$.

La definición de muerte se pregunta por el «qué», a un nivel básicamente conceptual, abstracto y filosófico, que no sirve en la práctica para determinar si un individuo ha muerto. Por ejemplo, la muerte es «cuando cesa de funcionar el organismo como un todo» (muerte clínica), o «cuando ocurre la muerte de todas las células del organismo»(muerte biológica), o «cuando el alma abandona el cuerpo» (muerte ontológica). Esta idea o concepto debe concretarse en una definición operativa que interpreta cuándo y cómo la idea se hace realidad en un caso concreto; por ejemplo: el cese irreversible del flujo de los fluidos corporales vitales», o el cese irreversible de las funciones encefálicas» ${ }^{10}$.

Los criterios diagnósticos proporcionan los elementos objetivos que determinan si la muerte ha ocurrido de acuerdo a la definición que se ha utilizado. Por ejemplo, el cese de la circulación de los fluidos corporales se acredita con la ausencia de la función cardíaca y pulmonar, y por cese de las funciones encefálicas se entenderá la ausencia de función del cerebro entero, incluido el tronco cerebral.

Luego de establecer los criterios, es necesario seleccionar las pruebas y procedimientos diagnósticos que acreditarán que los criterios se han cumplido. Estas pruebas pueden ser solo un examen clínico (auscultación, exploración neurológica), o requerir técnicas que van más allá (electroencefalograma, monitoreo cardíaco), cuando el caso lo requiere. Aunque las pruebas y procedimientos diagnósticos pueden variar con el progreso científico y tecnológico, una definición correcta permanecerá siendo válida a medida que pasa el tiempo ${ }^{10}$.
Examinaremos a continuación el estado actual de las nuevas definiciones de muerte que surgen en las últimas tres o cuatro décadas, y que tienen su origen en la aplicación de nuevas tecnologías de sostén vital, en particular los ventiladores artificiales. Son dos los criterios o formulaciones de muerte que se aceptan hoy en día: la muerte cardiopulmonar o tradicional, y la muerte encefálica. Un tercer criterio, la muerte de la neocorteza o cerebro superior, no es aceptado en ningún estado ni sociedad, pero se analiza por su interés ético y la confusión que puede producir en la práctica médica actual. A pesar de los cambios, los criterios tradicionales de muerte continúan siendo válidos en la mayoría de las ocasiones.

Criterio de muerte cardiopulmonar. Es el criterio clásico o tradicional, utilizado desde tiempos inmemoriales. Se define como la cesación permanente del flujo de los fluidos corporales vitales, que se comprueba mediante la detención irreversible de la función cardíaca y respiratoria. El cese aislado de cualquiera de las dos funciones conlleva el cese de la otra e inevitablemente sigue el cese de la actividad encefálica, lo que se explica porque las células del cerebro son las más sensibles a la falta de oxígeno y son las primeras en sucumbir. La detención de la circulación y respiración no siempre es sinónimo de muerte, ya que hay un breve período en que la muerte clínica no se transforma aún en muerte biológica (lesión celular imeversible), intervalo durante el cual puede ser reversible con determinados procedimientos y bajo ciertas condiciones (técnicas de reanimación cardiopulmonar).

Los criterios para determinar la muerte cardiopulmonar fueron recogidos en el Informe de la Comisión del Presidente, que dice: «n individuo con cese irreversible de las funciones circulatoria y respiratoria está muerto. Este cese debe reconocerse mediante el examen clínico adecuado». Este planteamiento fue criticado por aunar en la misma definición dos criterios diferentes de muerte, el cardiopulmonar y el cerebral. Los críticos concluyen que la detención de las funciones cardiorrespiratorias funciona como prueba de muerte sólo debido a que ese cese produce el verdadero criterio de muerte, el cese irreversible de todas las funciones del cerebro ${ }^{11}$. 
Criterios diagnósticos de la muerte encefálica. La muerte encefálica se define como la abolición total e irreversible de la función de todo el cerebro, incluyendo el tronco cerebral. La declaración de muerte encefálica debe ser segura e inequívoca, para lo cual se requiere no solamente de una serie de pruebas neurológicas efectuadas cuidadosamente, sino también establecer la causa del coma, asegurar su irreversibilidad, la resolución del más mínimo signo neurológico conducente a error, el reconocimiento de posibles factores de confusión, la interpretación de hallazgos de neuroimagen y la realización de cualquier prueba confirmatoria que parezca necesaria $^{12}$.

La exploración neurológica clínica ha permanecido como el elemento básico y central para la determinación de la muerte del cerebro, lo que ha sido adoptado por la mayoría de los países. El examen clínico de pacientes presuntamente en muerte encefálica debe ser realizado de modo sistemático, con extrema precisión y rigurosidad; se ha sugerido el siguiente protocolo diagnóstico que debe proceder de manera ordenada a través de las siguientes etapas ${ }^{12,13}$ :

a) Establecer la causa del coma. Antes de proceder al examen clínico es imprescindible conocer la causa del coma mediante la historia y el análisis de neuroimágenes que documenten la presencia de lesiones estructurales suficientes para explicar el daño cerebral irreversible. La condición de irreversibilidad sólo debe declararse luego del agotamiento de los esfuerzos terapéuticos en un período de tiempo definido de observación.

b) Exclusiones. El primer paso en el diagnóstico de muerte encefálica es descartar algunas condiciones médicas que confunden y pueden ser causas de coma reversible: alteraciones electrolíticas, ácido-base y endocrinas severas, el síndrome de enclaustramiento, la hipotermia, el shock severo y la intoxicación con drogas (sedantes del sistema nervioso y agentes bloqueadores neuromusculares).

c) Exploración clínica. Un examen neurológico completo incluye la documentación del coma y su causa, la ausencia de reflejos troncoencefálicos y el test de apnea. Generalmente dos exámenes neurológicos separados por al menos $6 \mathrm{~h}$ son confirmatorios, pero en ocasiones en que el daño cerebral es obviamente irreparable, un examen puede ser suficiente.

d) Pruebas de confirmación. Las pruebas de confirmación son opcionales en adultos pero se recomiendan en niños menores de 1 año. Estas pruebas pueden ser útiles cuando el cuadro clínico es confuso y en algunos países son requeridas por ley. En la práctica se utilizan en aproximadamente dos tercios de las determinaciones de muerte encefálica. Las pruebas más comunes son el electroencefalograma, estudios de flujo sanguíneo cerebral y los potenciales evocados.

Próximo al concepto de muerte encefálica, pero diferente a él, es el criterio de muerte del tronco encefálico, inspirado, como hemos mencionado, en el Código del Reino Unido ${ }^{7}$. De acuerdo a este Código la muerte del tronco cerebral puede diagnosticarse en la cabecera del paciente, basado sólo en la exploración clínica, sin recurrir a investigaciones complementarias. Estos criterios de tronco hacen énfasis en la ausencia de reflejos troncoencefálicos y son muy estrictos en definir el test de apnea. El electroencefalograma sería una prueba irrelevante de acuerdo a estos criterios, ya que no aportaría ninguna información mayor que la evaluación clínica.

Estado vegetativo persistente o muerte de la neocorteza cerebral. La formulación de muerte neocortical o de la corteza superior propone definir la muerte como la pérdida de la función que es indispensable para la naturaleza humana: la conciencia ${ }^{14}$. La defensa de esta posición plantea que da pérdida irreversible de las funciones corticales superiores, la conciencia y las funciones cognitivas son necesarias y suficientes para diagnosticar la muerte $\aleph^{15}$.

El ejemplo paradigmático de este tipo de muerte es el denominado estado vegetativo persistente, caracterizado por la destrucción completa e irreversible de la corteza cerebral, sede de las facultades llamadas superiores, y la persistencia de las funciones puramente vegetativas y vitales. 
Son pacientes que se encuentran en lo que se denomina coma vigil, pueden mantener los ojos abiertos, el ciclo vigilia-sueño, reflejos de retirada al estímulo, y mantienen indemnes sus funciones vegetativas (respiración espontánea, se alimentan por sonda gástrica, orinan, defecan); pero, han perdido por completo y de modo irreversible la capacidad de relacionarse con su medio (no se comunican, no sienten, no experimentan dolor).

Actualmente existen grandes problemas conceptuales y de orden práctico para aceptar una definición de la muerte humana basada en la ausencia del contenido de la conciencia. Esta aceptación requerinía un cambio radical en la definición de muerte, desde una visión fisiológica a considerar funciones sicológicas en dicha definición. Además, la conciencia es por naturaleza subjetiva y las funciones cognitivas son difíciles de explorar. Por último, se desconoce el sustrato morfológico en que se asienta el contenido de la conciencia y no se cuenta con un grupo de criterios o pruebas confirmatorias del estado vegetativo persistente, siendo muy difícil precisar su imeversibilidad ${ }^{10}$.

Por tales razones, no es posible aceptar la muerte definida con un criterio neocortical hoy en día; sin embargo, el estado vegetativo persistente, otra creación de la medicina moderna, plantea problemas éticos y legales muy serios en cuanto a la mantención o suspensión de tratamientos, no solamente de sostén vital sino también de hidratación y nutrición.

\section{CRITERIOS DE MUERTE: UNA REFLEXIÓN CRÍTICA}

La muerte es la cesación de la vida. Siendo la vida esencialmente organización, la muerte, que es su contrario, será en esencia desorganización o desestructuración. Definiendo la muerte como la pérdida de esta organización, o el cese del funcionamiento del organismo como un todo, nos parece que la muerte completa del cerebro (muerte encefálica), representa mejor esta definición que el cese de la circulación y respiración. El encéfalo en su totalidad es responsable de la integración del organismo como un todo, y esta función integradora es más compleja que la conexión circulatoria reproducible en una preparación artificial de órganos perfundidos. Nadie diría que un decapitado está vivo, pero en ellos el corazón puede seguir latiendo por un tiempo y sería teóricamente posible restablecer su circulación y respiración artificialmente.

Por otra parte, cuando se detiene la circulación y la respiración, la muerte no se produce de inmediato; hay un lapso de tiempo en que es posible revertir estas funciones vitales (reanimación cardiopulmonar), y si no se han producido lesiones celulares, el individuo puede seguir viviendo. ¿Qué produce la irreversibilidad de la muerte cardiopulmonar? La destrucción por anoxia de las células más sensibles, las del cerebro, a este daño. Por lo tanto, aun en este criterio de muerte, la muerte definitiva es la destrucción irreversible del encéfalo.

Por último, se ha argumentado que la función nutricional es lo más propio de la vida. Esto puede ser válido para seres unicelulares o preparaciones de tejido o un órgano, que pueden ser perfundidas y nutridas, pero no son un organismo complejo. Son sólo partes vivas de un organismo mantenidas en forma artificial. Todos los entes vivos requieren la capacidad de nutrición y respiración celular, pero no todos los que se nutren son organismos.

\section{LA MUERTE ENCEFÁLICA Y LA LEY}

Siendo una materia de tanta relevancia para cada individuo y la comunidad, no es extraño que la muerte y sus implicancias de diverso orden estén reguladas por la ley. Las legislaciones de distintos países varían porque éste es un tema que se enfrenta de modo diferente en sociedades con una amplia gama de diversidad cultural y religiosa que se expresa en la ley.

La legislación chilena mediante la ley $19.451^{16}$, vigente desde 1996, establece normas sobre trasplante y donación de órganos. Esta nueva ley, de difícil gestación, perfeccionó las disposiciones del Código Sanitario respecto a este tema, que databa desde 1983. Respecto al diagnóstico de muerte, esta ley significó algunos avances y aportes 
significativos, pero también quedaron algunos tópicos deficientes y no bien definidos:

Aportes de la Ley 19.451:

a) Define la muerte siguiendo criterios neurológicos (muerte encefálica), incorporando protocolos diagnósticos actualizados que establecen claramente las condiciones de acreditación de la muerte y sus exclusiones.

b) Permite realizar el diagnóstico de muerte encefálica sin pruebas instrumentales.

c) Incluye criterios diagnósticos específicos para la población pediátrica.

d) Permite conocer, en forma más amplia, la voluntad de donación en vida, ya sea como iniciativa personal, al acudir a una Notaría 0 al momento de internarse en un hospital 0 clínica, o como respuesta a una consulta formulada al momento de renovar la cédula de identidad o la licencia de conducir.

Deficiencias de la Ley 19.451:

No dilucida la ambigüedad de que el concepto de muerte encefálica y sus consecuencias (suspensión de tecnologías de sostén vital), sólo se pueden aplicar para los efectos de esta ley, es decir trasplantes y donación de órganos, y no en otra circunstancia. Esto es difícil de sostener porque no es posible estar muerto para unos efectos y para otros no. Es un notable vacío en la legislación chilena y la de otros países, que esta ley no corrigió.

\section{MueRTE ENCEFÁLCA, BIOÉTICA Y TRASPLANTE DE ÓRGANOS}

La historia de la bioética comparte con la de los trasplantes de órganos algunas coincidencias, como un nacimiento casi simultáneo y una evolución prácticamente paralela. La razón de esto es que el nacimiento de la bioética es consecuencia y una respuesta al rápido y sorprendente desarrollo de las ciencias biomédicas, una auténtica revolución, durante la segunda mitad del siglo XX. Surgidos en esta época y formando parte de dicha revolución biomédica, los trasplantes de órganos se han convertido en un procedimiento terapéutico aceptado, consolidado e imprescindible para muchas enfermedades de la medicina actual ${ }^{17}$.

En el trasplante de órganos convergen prácticamente la totalidad de los problemas éticos de la medicina, por lo que se lo ha llegado a considerar un micromodelo bioético, donde se adelanta el debate y la resolución de los problemas, aplicándose luego en otros campos de la medicina. Siguiendo la historia de los trasplantes se asiste al planteamiento sucesivo de diversos problemas éticos. El primer gran tema de debate, en los inicios del trasplante, década de los 50, fue la mutilación que exigía la donación de vivo. Resolviéndose este problema, surgió otro, el de los trasplantes en seres vivos con fines experimentales, que se desarrolla en la década de los 60, cuando se realizan los primeros trasplantes de casi todos los órganos sólidos. En la década de los 70, considerados los trasplantes ya terapéuticos, se plantea el problema de la donación de cadáver y una nueva definición de la muerte. La mayor disponibilidad de órganos de donante cadáver y el advenimiento de nuevos inmunosupresores, planteó en los 80, el problema de la distribución equitativa de órganos considerando que siempre serán un recurso escaso; aparejado a esto vino todo lo relacionado al comercio de órganos. En la década del 90 ha surgido como tema el de la compleja organización que se requiere para que la donación y los trasplantes funcionen de manera óptima. No es difícil avizorar que en los próximos años surgirán nuevas cuestiones éticas, como el trasplante de órganos procedentes de animales ${ }^{17}$.

Muchos principios éticos están en juego y fundamentan la medicina del trasplante y donación de órganos ${ }^{18}$. Por ejemplo, el principio de autonomía, de no usar al ser humano con una concepción instrumental, por más loables que sean los fines; también consecuencia de la autonomía será el énfasis en la libertad de las personas involucradas (donante y receptor) que se expresa en el consentimiento informado. Los principios de beneficiencia y no-maleficiencia tienen su lugar en la exigencia de seguridad de los procedimientos. La exigencia ética de la beneficiencia se concretaría en un llamado a la solidaridad humana como principio rector de toda la problemática de los trasplantes. 
Puesto en perspectiva, entonces, la definición y criterios de muerte encefálica es, por relevante que sea, solamente uno de los problemas éticos que el trasplante de órganos ha planteado y lo seguirá haciendo en los próximos años.

\section{MuERTE ENCEFÁLICA, TEMORES Y CONFIANZA PÚBLICA Y DIVERSIDAD CULTURAL-RELIGIOSA}

La imagen del paciente que muere en su casa rodeado del afecto de su familia ya casi no existe; es poco probable morir de esta manera en el mundo de hoy. Al intervenir y hacer confusa la frontera entre la vida y la muerte, la medicina moderna ha obligado a las personas y a la comunidad en general a enfrentar y definirse frente a la muerte como nunca antes. Por una parte, desde la introducción de las tecnologías de sostén vital en la medicina crítica, aparecieron temores de la aplicación no juiciosa y desproporcionada de estas tecnologías. Nacía el concepto del «ensañamiento terapéutico»y la prolongación artificial de la vida biológica, que debían ser evitados. De hecho, la muerte encefálica y el estado neurovegetativo persistente son creaciones de las tecnologías de sostén. Por otro lado, el desarrollo de la medicina del trasplante y donación de órganos provocan miedo y desconfianza en los familiares de que la declaración de la muerte de su ser querido pueda ser insegura y apresurada para beneficiar a otros. Los temores de la mutilación de cadáveres también aparecen relacionados con el trasplante de órganos.

Los temores y recelos acerca de la muerte encefálica provienen principalmente de que no es un concepto fácil de entender para la gente común. Intuitivamente a cualquier persona le costará comprender que un individuo pueda estar muerto si aún su corazón late y parece vivo. Los médicos tampoco han contribuido mucho a disipar estos temores cuando explican a la familia que «a su hijo se le ha diagnosticado muerte cerebral y morirá tan pronto le retiremos el respirador que lo mantiene vivo».

Sin embargo, la sociedad debe asumir estas dudas y temores de la gente porque no es posible revertir el avance científico-tecnológico; si a la comunidad le interesa y tiene el derecho de usufructuar del progreso tecnológico que ha mejorado sus condiciones de vida, también debe asumir sus consecuencias. La redefinición de la muerte es una de estas consecuencias, una de las más trascendentales del siglo pasado. Todo cambio de esta magnitud en un tema tan delicado necesita muchos años, tal vez generaciones para incorporarse plenamente a la cultura social. Los individuos viven, se desarrollan y se educan en sociedades marcadas por la diversidad cultural y religiosa, por lo tanto la penetración de los cambios es a distinta velocidad en estas sociedades.

A modo de ejemplos, se puede señalar que en Japón el concepto de muerte cerebral no fue aceptado en la legislación hasta 1997. La única cita bibliográfica del informe de Harvard sobre muerte cerebral da cuenta de la posición de la Iglesia Católica de la época, a través del Papa Pío XII, quien ya en 1958 declaraba que la prolongación de la vida por medios extraordinarios en los pacientes críticos y la verificación del momento de la muerte eran de incumbencia estrictamente médica ${ }^{18}$. En la actualidad, Juan Pablo II también ha reconocido en diversos documentos la realidad del criterio neurológico de muerte, y ha declarado que aplicados rigurosamente, los criterios de muerte encefálica no parecen en conflicto con una correcta concepción antropológica ${ }^{19}$. El planteamiento de las grandes religiones equipara el de la iglesia católica. La Ley Judía reconoce el valor ético de la donación de órganos, pero exige que los cadáveres sean tratados con respeto. El Código Islámico de ética médica aprobó en 1981 la donación de órganos como un beneficio a la sociedad y destaca que ssi los vivos pueden donar, más aún los muertos, ningún daño se inflige al cadáver si se toman los órganos para hacer un buen uso en una persona viva. Esto es, sin duda, caridad $\gg^{8}$.

\section{Conclusiones}

La perspectiva histórica nos mostró el escenario de un concepto de muerte siempre en evolución desde la antigüedad hasta nuestros días. Rodeada 
de incertidumbre, la determinación de la muerte no fue una tarea fácil en muchas épocas de la humanidad. Winslow, un anatomista, ya había dicho en 1742: muerte cierta, muerte incierta; es completamente cierto que hemos de morir, pero es incierto saber a veces si alguien está muerto». En nuestra época afirma Pallis que puede haber varias formas de morir, pero sólo hay una forma de estar muerto »1.

Al finalizar este artículo, debemos insistir que las «arias formas de morin han sido una consecuencia de la revolución biomédica y tecnológica que irrumpe en nuestra sociedad en la segunda mitad del siglo XX. La sociedad debe asumir estos cambios con todas sus implicaciones éticas, médicas, culturales y legales, porque son irreversibles.

Se han revisado las definiciones y criterios de muerte aceptados hoy, concluyendo que la muerte encefálica, basada en criterios neurológicos, cumple mejor los requisitos de «ese permanente del funcionamiento del organismo como un todo», que subyace la definición de muerte en general. El diagnóstico de muerte encefálica está basado en un protocolo neurológico clínico establecido, sim-

\section{REFERENCIAS}

1. Rodríguez del Pozo P. La determinación de la muerte: historia de una incertidumbre. Jano 1993; XLIV: 71-77.

2. Gracia D. Etica de los confines de la vida. Editorial Códice, Santa Fe, Colombia. 1998.

3. Wertheimer P, Jouvet M, Descotes J. A propos du diagnostic de la mort du système nerveux dans les comes avec arrêt respiratoire traités par respiration artificielle. Press Med 1959; 67: 87-8.

4. Molaret P, Goulon M. Le coma depassé (memoire preliminaire). Rev Neurol (Paris) 1959; 101: 3-5.

5. A definition of irreversible coma: report of the $\mathrm{Ad}$ Hoc Committee of the Harvard Medical School to examine the definition of brain death. JAMA 1968; 205: 337-40.

6. Mohandas A, Chou SN. Brain death: a clinical and pathological study. J Neurosurg 1971; 35: 211-8.

7. Diagnosis of brain death: statement issued by the honorary secretary of the Conference of Medical ple y seguro que, seguido con prudencia, rigurosidad y precisión, es inequívoco e infalible.

Los fundamentos éticos de la medicina del trasplante y la donación de órganos, realizada principalmente en personas en muerte encefálica, fueron examinados. Habiéndose consolidado hoy como una terapia universalmente aceptada que beneficia a mucha gente, el trasplante de órganos descansa en una base ética sólida, principalmente en los principios de autonomía, beneficiencia y no-maleficiencia.

El recelo y la desconfianza social, y de algunos sectores de la comunidad médica, hacia la muerte encefálica y la donación de órganos son comprensibles, porque es una situación relativamente nueva, y que necesita mayor educación. Las diversas sociedades, legislaciones, culturas y religiones han ido aceptando gradualmente la evolución del concepto de muerte, lo que refleja la solidez en que estos cambios se fundamentan. Solo una educación permanente a la sociedad en general y a la comunidad médica en particular, ayudará a despejar totalmente las dudas, incertidumbres y desconfianzas que estos temas aún despiertan.

Royal Colleges and their Faculties in the United Kingdom on 11 October 1976. BMJ 1976; 2: 11878.

8. Guidelines for the determination of death. Report of the medical consultants on the diagnosis of death to the President's Commission for the study of ethical problems in medicine and biomedical and behavioral research. JAMA 1981; 246: 2184-6.

9. Bernat JL, Culvert CM, Gert B. On the definition and criterion of death. Ann Intern Medicine 1981; 94: 389-94.

10. Escalante JL La definición de muerte. Trasplantes de órganos: problemas técnicos, éticos y legales. Javier Gafo (editor); 1996; 53-74.

11. Paшis C. Brainsteam death, en Braakman R (ed.) Handbook of clinical neurology: Head injury, Amsterdam: Elsevier Science Publishers BV 1990; 13: 441-96.

12. WijDICKs EFM. The diagnosis of brain death. N Engl J Med 2001; 344: 1215-21. 
13. Escudero D, Otero J. Avances clínicos y legales en el diagnóstico de muerte encefálica durante la década de los trasplantes en España. Nefrología 2001; Vol XXI. Suplemento 4.

14. Truog RD, Flacker JC. Rethinking brain death. Crit Care Med 1992; 20: 1705-13.

15. YoungNER SJ, BARTLETT ET. Human death and high technology: The failure of the wholebrain formulations. Ann Intern Med 1983; 99: 252-8.

16. Ley № 19.451. Establece normas sobre trasplante y donación de órganos. República de Chile.
Publicada en el Diario Oficial con fecha 10 de abril, 1996.

17. GRACIA D. Trasplante de órganos: medio siglo de reflexión ética. Nefrología 2001; Vol XXI. Suplemento 4. 13-29.

18. GAFO J. Reflexiones éticas sobre los trasplantes de órganos. Trasplantes de órganos: problemas técnicos, éticos y legales. Javier Gafo editor 1996; 137-60.

19. Juan Pablo II. Discurso inaugural del XVIII Congreso Internacional de la Sociedad de Trasplante. Roma, 29 de agosto, 2000. 